\title{
Network search method in the design of extreme ultraviolet lithographic objectives
}

\author{
Oana Marinescu* and Florian Bociort \\ Optics Research Group, Delft University of Technology, Lorentzweg 1, 2628 CJ, Delft, The Netherlands \\ ${ }^{*}$ Corresponding author: o.marinescu@gmail.com \\ Received 16 July 2007; revised 14 October 2007; accepted 16 October 2007; \\ posted 18 October 2007 (Doc. ID 83335); published 3 December 2007
}

\begin{abstract}
The merit function space of mirror system for extreme ultraviolet (EUV) lithography is studied. Local minima situated in the multidimensional optical merit function space are connected via links that contain saddle points and form a network. We present networks for EUV lithographic objective designs and discuss how these networks change when control parameters, such as aperture and field, are varied, and constraints are used to limit the variation domain of the variables. A good solution in a network, obtained with a limited number of variables, has been locally optimized with all variables to meet practical requirements. (C) 2007 Optical Society of America

OCIS codes: $\quad 220.0220,220.2740,220.3740,220.4830,220.3620$.
\end{abstract}

\section{Introduction}

Finding good solutions in multidimensional merit function spaces is a difficult task, because of the large number of existing local minima. Commercial optical design programs contain, nowadays, powerful global optimization algorithms, such as global synthesis [1-4], global explorer [5], simulated annealing [6], and genetic algorithms [7]. However, these algorithms give solutions as single points in the merit function space, without information about any relations between them.

It has been shown [8] that the local minima situated in the multidimensional optical merit function space are connected via optimization paths that start from a special type of saddle point (saddle point with Morse index 1) and form a network. Examples of the network structure for simple systems (Double Gauss, Cooke Triplet) have also been given [8-11]. Extremeultraviolet (EUV) lithography [12-16] is a future generation lithography technique that uses radiation with a wavelength of 10 to $14 \mathrm{~nm}$ to print microchips with feature sizes below $30 \mathrm{~nm}$. We give examples of networks for the merit function space of mirror designs for EUV lithography.

0003-6935/07/358385-09\$15.00/0

(C) 2007 Optical Society of America
We first give a brief description of the network search method for finding good local minima [9]. Then, we present network graphs for the merit function space of a six-mirror EUV design problem with only six variables. We discuss how variations of the control parameters, such as numerical aperture and field, and changes of the constraints influence these networks. A good six-mirror configuration, generated by our network search method, is then locally optimized for practical demands using all the variables.

\section{Saddle Points and Networks}

In the neighborhood of a critical point (i.e., a point at which the gradient of $F$ vanishes), the merit function $F$ is given by

$$
F\left(x_{1}, x_{2}, \ldots, x_{N}\right)=F_{0}+\sum H_{i j} x_{i} x_{j},
$$

where $F_{0}$ is the value of $F$ at the critical point and $x_{i}$ are the (shifted) optimization variables. (The origin of the coordinate system is placed at the critical point.) At a critical point, for which the determinant of the matrix

$$
H_{i j}=\frac{1}{2} \frac{\partial^{2} F}{\partial x_{i} \partial x_{j}}
$$

is nonzero, the coordinate system can be rotated so that Eq. (1) contains only squares of the new variables 


$$
F\left(\bar{x}_{1}, \bar{x}_{2}, \ldots, \bar{x}_{N}\right)=F_{0}+\sum \lambda_{i} \bar{x}_{i}^{2},
$$

where $\lambda_{i}$ are the eigenvalues of the matrix $H_{i j}$ [the barred new variables in Eq. (3) are measured along the eigenvectors of $H_{i j}$.

In topology, the number of negative eigenvalues in Eq. (3) is called the Morse index of the critical point. A negative eigenvalue means that, along the corresponding eigenvector the critical point is a maximum, and a positive eigenvalue indicates a minimum along the corresponding eigenvector direction. Local minima have a Morse index (MI) of 0 , local maxima have a MI of $N$ (the number of variables) and saddle points have a MI between 1 and $N-1$.

For finding the network structure it is sufficient to consider only saddle points with $\mathrm{MI}=1$, i.e., saddle points that are maxima along one direction [9]. In two dimensions, all saddle points have $\mathrm{MI}=1$ and resemble a horse saddle. In variable spaces with a higher dimensionality, the saddle points with MI = 1 are those which are similar to twodimensional saddle points: the merit function decreases on the two sides along the unique maximum direction. The central element in finding a network structure is the detection of saddle points with MI $=1$ around known local minima. Saddle point detection algorithms that have certain similarities with the one described below are used to study the energy landscape of systems with many atoms [17-19].

We start at an arbitrary minimum. To detect saddle points with $\mathrm{MI}=1$ around it, a set of directions is defined, each characterized by a unit direction vector $\mathbf{s}=\left(s_{1}, s_{2}, \ldots, s_{N}\right)$. In the implementation of the method used in this work, these directions are those of the eigenvectors of $H_{i j}$, computed numerically at the given minimum. Previously [11], we have described in full detail our method to compute all these eigenvectors by using the local optimization capability of optical design software. It follows from Eq. (3) that, around local minima, the surfaces along which the merit function is constant have the shape of ellipsoids. The eigenvectors at $H_{i j}$ correspond to the directions of the half-axes. A set of hyperplanes or-

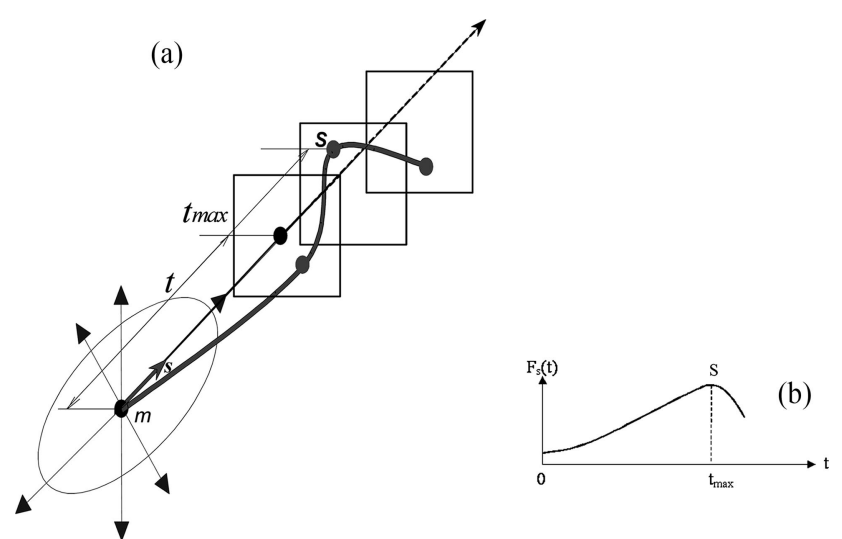

Fig. 1. Illustration of the saddle point detection algorithm: (a) constraint local minimization in a set of hyperplanes orthogonal to $\mathbf{s}$; (b) one-dimensional maximization along $\mathbf{s}$.

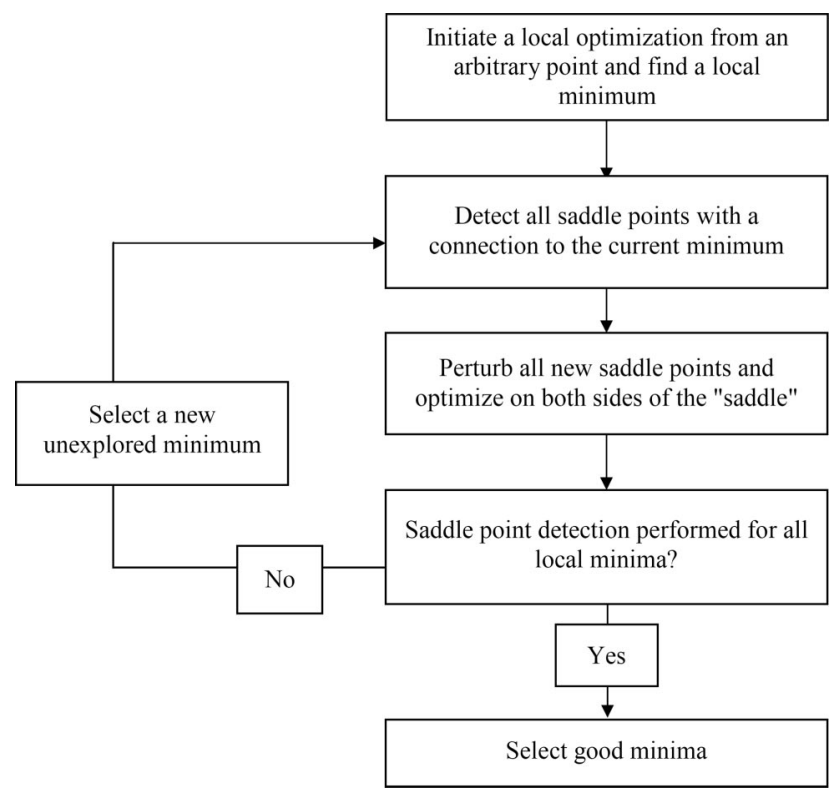

Fig. 2. Flow chart for the network search method.

thogonal to a given direction $\mathbf{s}$ is selected:

$$
s_{1} x_{1}+s_{2} x_{2}+\cdots+s_{N} x_{N}=t
$$

where $t$ is the distance between the local minimum and the hyperplane [see Fig. 1(a)]. For a given value of $t$, the saddle point detection algorithm computes the local minimum of $F$ in the hyperplane defined by Eq. (4). The curved line in Fig. 1(a) shows symbolically the position of the constraint minimum as a function of $t$. The merit function value at the constraint minimum is denoted by $F_{s}(t)$. We start at the local minimum (where $t=0$ ) and then increase $t$ gradually. The merit function $F_{s}(t)$ increases at the beginning [Fig. 1(b)]. At a certain $t=t_{\max }, F_{s}(t)$ reaches a maximum value (point $S$ ), after which it decreases. The constraint minimum at $t=t_{\max }$ is then a saddle point with $\mathrm{MI}=1$.

From such a saddle point, two distinct local minima are obtained by letting the optimization go down on the two sides of the saddle. (All eigenvector directions are tried out, and for a direction $\mathbf{s}$ that has led to successful saddle point detection, one of these two minima is the one that has been used for detecting the saddle point.) The optimization paths, together with the saddle point with $\mathrm{MI}=1$, form a link in the

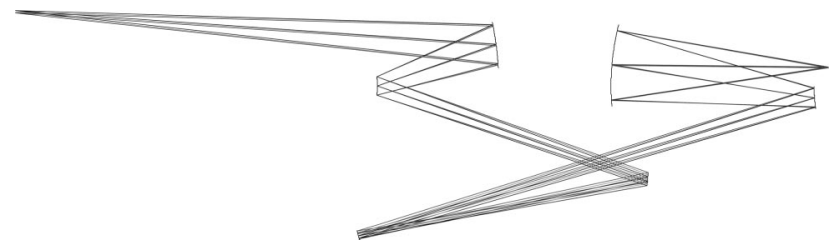

Fig. 3. Six-mirror microlithographic projection system with object heights between 114 and $118 \mathrm{~mm}$, a numerical aperture of 0.16 , and a magnification of 0.25 . The aperture stop is placed at the second surface. 
optimization space between the two minima. The local minima that are connected together in this way form a network.

A network search method based on saddle-point detection has been developed [8-11] (see Fig. 2). For a given local minimum, the algorithm attempts to detect all saddle points with MI $=1$ that are connected with it. Via local optimization performed on the other side of each saddle point, new minima are obtained. At each new minimum a saddle point search is performed to find the remaining part of the network. At the last stage the best solutions (i.e., the solutions for which the imaging quality is satisfactory) are selected.
The algorithm has been implemented in our program NETMIN, which for local optimization calls the commercial optical design program CODE $\mathrm{v}$ [20]. The two programs communicate via the operating system.

Finding saddle points is computationally more expensive than finding local minima. Therefore, the network search method can be successfully applied if the number of local minima is not excessively large.

\section{Topography of the Merit Function Space of Extreme Ultraviolet Mirror Systems}

We have used the algorithm described above on several EUV ring-field configurations that consist of four, six, or eight mirrors. The patent literature shows that

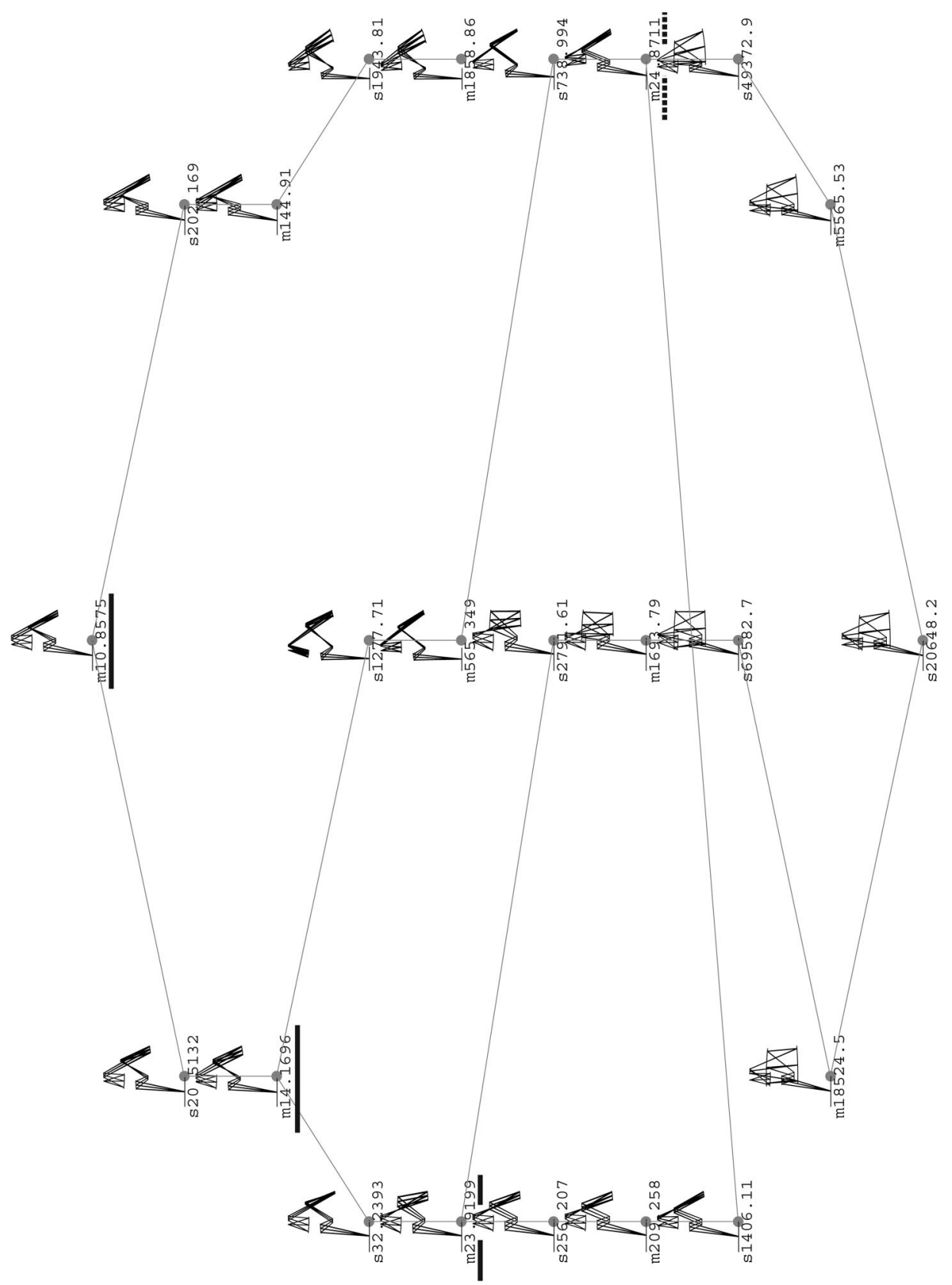

Fig. 4. Network structure of a six-mirror system search with the specifications of the system shown in Fig. 3, where $s$ represents saddle points, and $m$ represents minima. The value of the merit function is also shown. 
these types of system are of significant interest [21-29]. We present results of the network search for a six-mirror configuration. (A system with the specifications that have been used is shown in Fig. 3.) Since, for EUV systems, in certain domains of the merit function space even local optimization tends to become unstable [30], we did not attempt to detect the entire network, but we focused on regions of interest containing stable domains of the merit function space, i.e., the domains in which the systems continue to exist when changes of the optimization constraints or the control parameters (numerical aperture and field) are made, as shown in what follows. All surfaces are aspheric, with aspheric coefficients going up to the tenth order on each surface. However, because network explorations with all vari- ables (37 in this case: six curvatures, 24 aspheric coefficients, and seven distances between surfaces) is very time consuming, in all our searches only the six surface curvatures have been used as variables, while the aspheric coefficients have been kept constant with nonzero values. (Curvature changes generate much more local minima than changes of distances and aspheric coefficients.) The default CODE $\mathrm{v}$ merit function, which is based on transverse aberrations, has been used.

In our research, we followed two directions. We analyzed the change in the merit function space when

(a) the control parameters, numerical aperture, and field are modified;

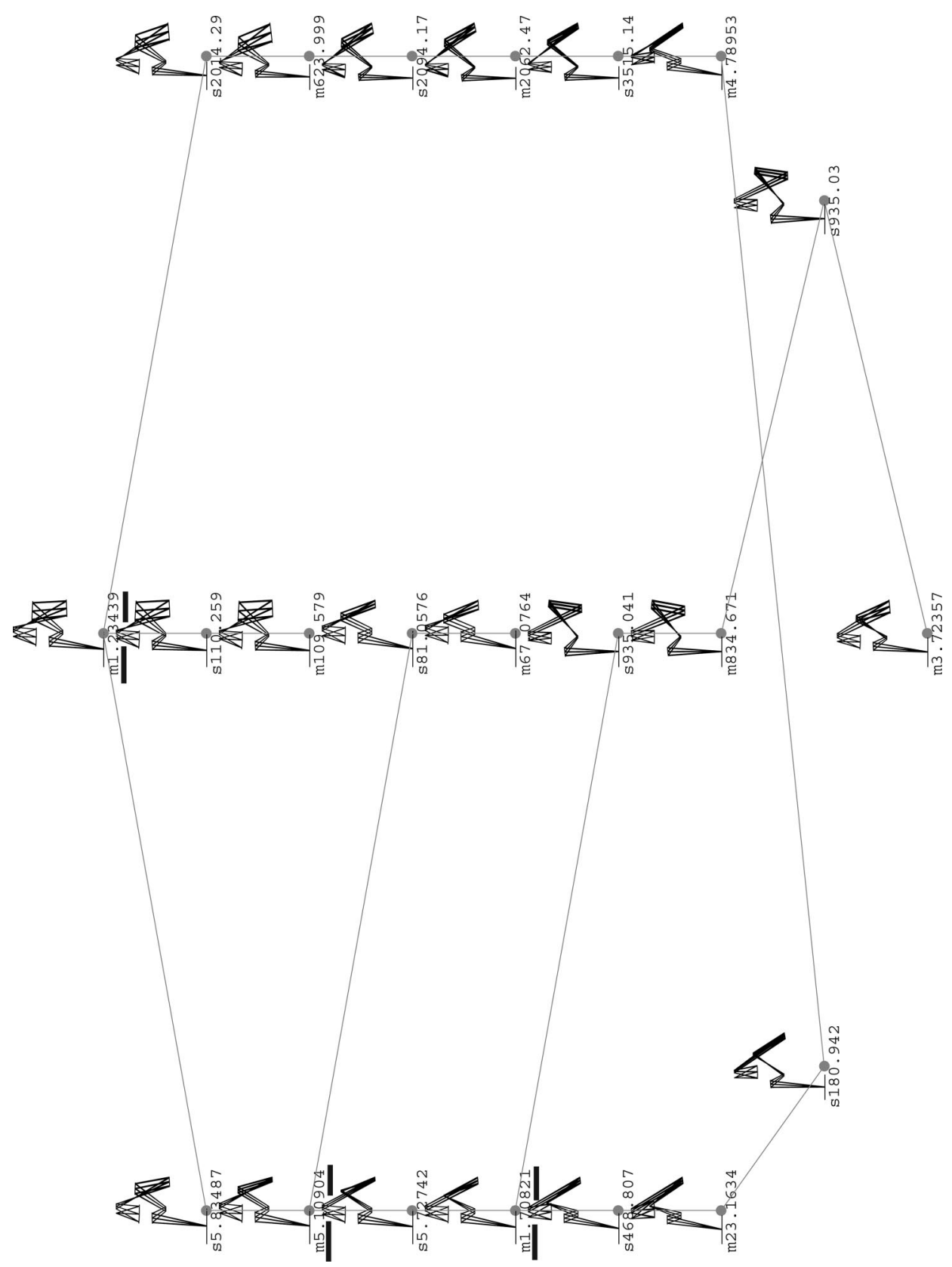

Fig. 5. Network structure at reduced numerical aperture and field. 
(b) at fixed values of numerical aperture and field, constraints (telecentricity at the image plane, quasitelecentricity at the object plane, freedom of obstruction, and distortion within certain limits) are used to limit the variation domain of the variables.

A. Influence of the Control Parameters on the Merit Function Space of Extreme Ultraviolet Mirror Systems

In our first example, we discuss the topography of the merit function space corresponding to the six-mirror system presented in Fig. 3. The system has been constrained to be paraxially telecentric on the image side, i.e., the paraxial chief ray leaving the last mirror must be parallel to the optical axis. The magnification was kept constant at a value of 0.25 . A region of interest in the network, as detected with the present version of NETMIN, is presented in Fig. 4. In this graph each node represents either a local minimum or a saddle point with $\mathrm{MI}=1$ in the merit function space, and the lines connecting the nodes show to which minima the two optimization paths that start at saddle points should go. This graph allows us to determine the relationships between different local minima independently of the dimensionality of the merit function space.

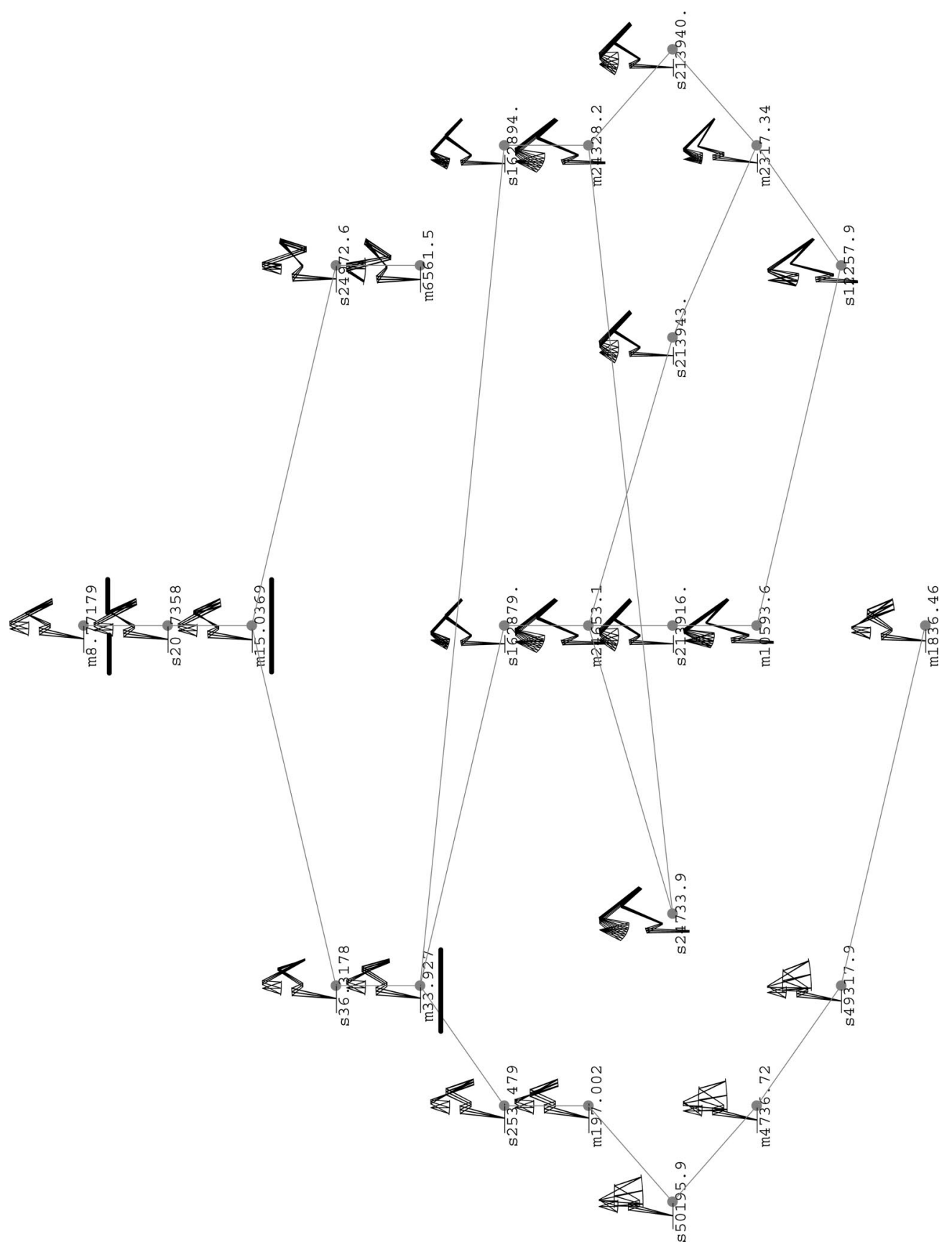

Fig. 6. Network structure with real telecentricity requirement in the image space. 
The part of the network presented in Fig. 4 provides useful information. For example, we observe that, in addition to the minimum with the lowest merit function ( $m 10.8575)$, there are two other solutions in this space that have low values of the merit function. These two solutions and the best minimum are underlined with solid lines in Fig. 4. The three solutions are connected via saddle points that have slightly higher values of the merit function. Therefore, along the entire network path between these three solutions in the merit function space, the variations of the merit function are small compared to the variations in the rest of the merit function landscape.

The connections between the best systems in the network, and the fact that outside the region where they were found the merit function increases drastically, are consistent with Isshiki's conjecture according to which the minima with nearly the lowest merit function value are situated in a "string winding through the merit function space" [31].

Analyzing the configurations situated outside the network path connecting the best three solutions, we observe that many of them are situated either in an obstructed space (at this stage freedom of obstruction has not been imposed as constraint) or in a space where they are not real telecentric at the wafer side (i.e., because of aberrations, the real chief ray deviates from the paraxial one and is not parallel to the optical axis). As mentioned above, here we have imposed the paraxial telecentricity constraint. However, if the freedom of obstruction is also imposed as constraint during the network search, some previously obstructed local minima, e.g., minimum $m 24.8711$ (underlined with a dotted line), become unobstructed. Typically, a slight obstruction can be corrected individually in later design stages, if necessary. A similar approach is successful when a violation of the real telecentricity requirement is encountered.

When examining the network link by link, one observes that the system shapes change gradually. For instance, on the path starting at $m 24.8711$ (dotted line), that goes via $s 1406.11$ (on the left side of the drawing), $m 209.258$ and $s 256.207$ to $m 23.9199$ (solid line), we observe how a succession of small changes accumulate. Whereas the first configuration in this sequence has a slight obstruction (caused by the position of the third mirror), the last configuration is free of obstruction (the third mirror has shifted below the rays reflected by the fourth mirror and collected by the fifth one). Local minimum $m 23.9199$ has a modified new configuration. The same applies for the path from $m 24.8711$ to $m 14.1696$ (solid line) via $s 738.994, m 565.349$ and $s 1277.71$.

The results obtained by NETMIN and shown in Fig. 4 have been compared with those of GLOBAL SYNTHESIS, the algorithm included in the commercial optical design program CODE V. The same optimization conditions have been used as in Fig. 4. All solutions found by GLOBAL SYNTHESIS have also been found by NETMIN. Both programs have found the three good solutions (solid line).
When we reduce both the numerical aperture and the field by one third, our algorithm finds the network shown in Fig. 5. Again, three solutions (underlined in Fig. 5) having the best performance are observed. If we reset the numerical aperture and the field of these three solutions to their initial values and then we locally reoptimize them, we observe that they converge to the three best solutions in Fig. 4: $m 1.23439$ becomes $m 23.9199, m 5.10904$ becomes $m 14.1696$, and $m 1.70821$ becomes $m 10.8575$. Note that the best minimum at lower numerical aperture and field ( $m 1.23439$ ) is not the one that becomes the best minimum at the original values of these control parameters. However, it still corresponds to one of the good solutions in Fig. 4.

We made several network runs for the six-mirror system with different values of the control parameters. Comparing the results, we observed that small changes in the control parameters do not change the networks significantly. However, especially when the changes are large, parts of the networks tend to appear or disappear. For instance, two minima and a saddle point in between $[m 1.23439, s 110.259$, and $m 109.579$ in (Fig. 5)] found at low numerical aperture and field become a single minimum [ $m 23.9199$ in (Fig. 4)] when the values of the two parameters are increased. The network structure (i.e., the number of nodes and the connections between them) changes, but the best solutions remain in the structurally stable part of the network. In fact, similar behavior is observed for many other types of system as well. For instance, for the Cooke Triplet, if the aperture is low enough, we have two minima having the well known Cooke Triplet shape and a saddle point between them, but when the aperture is increased beyond a threshold value, only one minimum remains [11].

\section{B. Influence of Constraints on the Merit Function Space of Extreme Ultraviolet Mirror Systems}

We also analyzed the merit function space of the same six-mirror system (Fig. 3), but with different constraints. First, we replaced the paraxial telecentricity with a real telecentricity requirement, (i.e., the real chief ray at the wafer stage must be parallel to the optical axis).

Despite significant differences in the network (Fig. 6), (which are either real or are caused by the limitations of the present version of NETMIN), the three good solutions mentioned earlier are found again. When

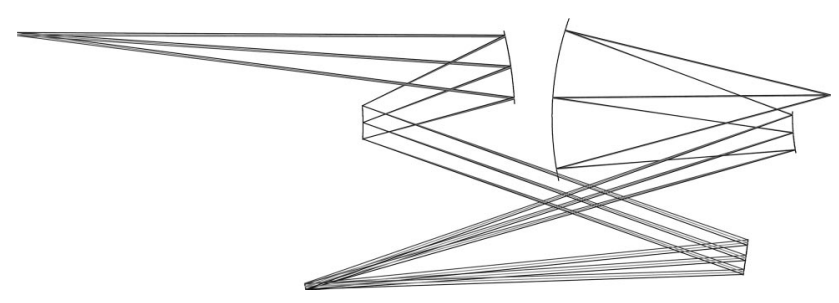

Fig. 7. Optimized six-mirror projection system for EUV lithography. The aperture stop is situated at the second mirror. The system is unobstructed. At the wafer side the system is telecentric, i.e., the chief ray is (approximately) perpendicular on the image plane. 
Table 1. Specifications for the Optimized EUV Mirror System Presented in Fig. 7

\begin{tabular}{ll}
\hline \multicolumn{1}{c}{ Specifications } & \multicolumn{1}{c}{ Value } \\
\hline NA & 0.25 \\
Field & $28.5-29.5 \mathrm{~mm}$ \\
Wavelength & $13 \mathrm{~nm}$ \\
Magnification & 0.25 \\
Distortion & $<1 \mathrm{~nm}$ per field \\
Strehl ratio & $>0.986$ \\
RMS Wavefront aberration & $<16 \mathrm{~m} \lambda$ \\
\hline
\end{tabular}

reoptimizing them with paraxial telecentricity constraint at the image plane they converge to the three best solutions of the network detected under these conditions: the local minimum $m 15.0369$ (in Fig. 6) becomes $m 14.1696$ in (Fig. 4), and local minimum $m 33.927$ becomes $m 23.9199$. Interestingly, the best minimum $m 8.77179$ corresponds to the best minimum (in Fig. 4), $m 10.8575$.

The same sort of network analyses have also been made with other constraints: paraxial and real quasitelecentricity on the object side (i.e., the upper paraxial, respectively, real marginal ray must be parallel to the optical axis), freedom of obstruction, and distortion control. Because we had only six variables, we did not use all the constraints at the same time, but we used different combinations of them.

In addition to the merit function space of the system presented in Fig. 3, we studied the merit function space of a number of different six-mirror configurations, as well as for a four- and several eight-mirror systems [32]. In all cases, we have observed that, when constraints are modified, the same system remains the best one (i.e., after reoptimization the best system in one run becomes the best system in the other run). Surprisingly, this happens even when distortion is controlled (within limits that were not very tight), but we believe that this result is specific to our systems. For other types of system, distortion control may change the best system. We have compared the results obtained by our program NETMIN with those generated by GLOBAL SYNTHESIS. In virtually all cases, both programs detect the same good local minima.

\section{Fully Optimized Designs}

In order to satisfy practical requirements, the best minimum in Fig. 6 ( $m 8.77179)$ has been further im-

Table 2. Chief-Ray Angles of Incidence and the Angular Spread in the EUV Mirror System Shown in Fig. 7

\begin{tabular}{ccc}
\hline Mirror & Angle & Angular Spread \\
\hline 1 & $12.17^{\circ}$ & $0.93^{\circ}$ \\
2 & $20^{\circ}$ & $2.75^{\circ}$ \\
3 & $11.62^{\circ}$ & $0.98^{\circ}$ \\
4 & $6.88^{\circ}$ & $1.86^{\circ}$ \\
5 & $12.88^{\circ}$ & $6.96^{\circ}$ \\
6 & $4.39^{\circ}$ & $0.84^{\circ}$ \\
\hline
\end{tabular}

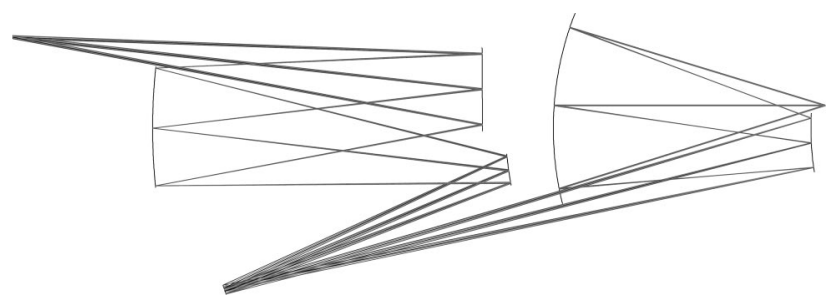

Fig. 8. 0.3 numerical aperture six-mirror projection system for EUV lithography.

proved with traditional design techniques, using all surface curvatures, distances between surfaces, and aspheric coefficients as optimization variables. The resulting design, shown in Fig. 7, has very good imaging qualities. Some of the system's specifications and performances are shown in Table 1. All surfaces are aspheric. The angle of incidence of the chief ray at the mask is $4.4^{\circ}$. For multilayer compatibility, the angular spread (i.e., the difference between the maximum and the minimum angle value) at each surface has been kept smaller than $7^{\circ}$ (see Table 2).

The numerical aperture of the system shown in Fig. 7 has been increased to 0.3. During the local optimization process, the freedom of obscuration constraint [33] was temporarily removed so that the third mirror can change its position. Further optimization with all required constraints of the new configuration leads to the six-mirror system shown in Fig. 8 (See Table 3. Full specifications can be found in [32].) The design requirements, in terms of distortion, telecentricity at the wafer, quasi-telecentricity at the mask, and freedom of obscuration, are satisfied. The angular spread of each surface is smaller than $8^{\circ}$. The rms wavefront aberration is smaller than $31 m \lambda$ (see Table 4).

Thus, from the same network system, used as the starting design configuration, we obtained two sixmirror systems with good imaging qualities: one with a wavefront aberration of $16 \mathrm{~m} \lambda$ at a numerical aperture of 0.25 , and the second one with a wavefront aberration of $31 \mathrm{m \lambda}$ at a numerical aperture of 0.3 . For the second configuration, the same rms wavefront aberration as for the first one $(16 m \lambda)$ is obtained at a value of the numerical aperture of 0.29 .

Table 3. Specifications and Performance of the Optimized EUV Mirror System Shown in Fig. 8

\begin{tabular}{ll}
\hline \multicolumn{1}{c}{ Specifications } & \multicolumn{1}{c}{ Value } \\
\hline NA & 0.3 \\
Field & $28.5-29.5 \mathrm{~mm}$ \\
Wavelength & $13.5 \mathrm{~nm}$ \\
Magnification & 0.25 \\
Distortion & $<1 \mathrm{~nm}$ \\
Strehl ratio & 0.944 \\
RMS Wavefront aberration & $<31 \mathrm{~m} \lambda$ \\
\hline
\end{tabular}


Table 4. Chief Ray Angles of Incidence for the EUV Mirror System Presented in Fig. 9

\begin{tabular}{ccc}
\hline Mirror & $\begin{array}{c}\text { Angle } \\
(\mathrm{deg})\end{array}$ & $\begin{array}{c}\text { Angular Spread } \\
(\mathrm{deg})\end{array}$ \\
\hline 1 & 6.42 & 4.26 \\
2 & 6.62 & 1.55 \\
3 & 14.63 & 4.59 \\
4 & 4.36 & 2.23 \\
5 & 11.03 & 7.86 \\
6 & 4.07 & 2.14 \\
\hline
\end{tabular}

\section{Conclusions}

We have illustrated the idea that local minima form a network with examples of EUV mirror systems, and we have shown how networks can be used to indicate good starting configurations for subsequent traditional design.

When control parameters are modified, the network structure changes, but the good systems tend to remain as nodes in a certain part of the network. We call such parts of the network stable regions. Present experience with EUV and other types of design suggests that systems from stable regions in the network are the candidates for further design. When, at constant values of the numerical aperture and field, constraints are modified, the same node in the network tends to remain the best minimum.

We have also shown that a relaxed use of constraints in the network search is sufficient for the purpose of generating starting points for further traditional local optimization. Moreover, we have shown that starting from a solution found by our network detection algorithm in a low-dimensional search, we have obtained high-quality six-mirror systems with performances that make them suitable for practical applications (a Strehl ratio larger than 0.986 at a numerical aperture of 0.25 , and a Strehl ratio larger than 0.944 at a numerical aperture of 0.3 ).

The projection system presented in Fig. 8 has a configuration similar to that used in existing EUV lithographic tools with a numerical aperture of 0.25 $[34,35]$. However, our result shows that high-quality imaging can be obtained at the higher numerical aperture of 0.3 as well.

O. Marinescu gratefully acknowledges the financial support from ASML Holding NV. We also thank Eco van Driel for programming NETMIN.

\section{References}

1. T. G. Kuper and T. I. Harris, "Global optimization for lens design—an emerging technology," Proc. SPIE 1780, 14-28 (1992).

2. T. Kuper and T. Harris, "A New Look at Global Optimization for Optical Design,” Photonics Spectra 151-160 (January 1992).

3. J. P. McGuire, Jr., "Designing easily manufactured lenses using a global method," Proc. SPIE 6342634200 (2006).

4. J. R. Rogers, "Using global synthesis to find toleranceinsensitive design forms," Proc. SPIE 6342 63420M (2006).

5. M. Isshiki, H. Ono, K. Hiraga, J. Ishikawa, and S. Nakadate,
"Lens design: global optimization with escape function," Opt. Rev. 6, 463-470 (1995).

6. G. W. Forbes and A. E. W. Jones, "Towards global optimization with adaptive simulated annealing," Proc. SPIE 1354, 144151 (1991)

7. K. E. Moore, "Algorithm for global optimization of optical systems based on genetic competition," Proc. SPIE 3780, 40-47 (1999).

8. F. Bociort, E. van Driel, and A. Serebriakov, "Networks of local minima in optical system optimization," Opt. Lett. 29, 189191 (2004)

9. E. van Driel, F. Bociort, and A. Serebriakov, "Topography of the merit function landscape in optical system design," Proc. SPIE 5249, 353-363 (2004).

10. F. Bociort, A. Serebriakov, and M. van Turnhout, "Saddle points in the merit function landscape of systems of thin lenses in contact," Proc. SPIE 5523, 174-184 (2004).

11. F. Bociort, E. van Driel, and A. Serebriakov, "Network structure of the set of local minima in optical system optimization," Proc. SPIE 5174, 26-34 (2003).

12. J. J. M. Braat, "Extreme UV lithography, "A candidate for next-generation lithography," Proc. SPIE 4016, 2-7 (2000).

13. K. Diefendorff, "Extreme lithography," Microprocessor Report 1-10 (Reed Elsevier, 19 June 2000).

14. S. A. Lerner, J. M. Sasian, and M. R. Descour, "Design approach and comparison of projection cameras for EUV lithography," Opt. Eng. 39, 792-802 (2000).

15. D. W. Sweeney, "Extreme ultraviolet lithography," in Encyclopedia of optical Engineering (Marcel Dekker, 2003), pp. 485491.

16. H. J. Levinson and W. H. Arnold, Handbook of Microlithography, Micromachining, and Microfabrication, Vol. PM39 of SPIE Press Monograph Series (SPIE Press, 1997), pp. 11-126.

17. G. T. Barkema and N. Mousseau, "Event-based relaxation of continuous disordered systems," Phys. Rev. Lett. 77, 43584361 (1996).

18. N. Mousscau and G. T. Barkema, "Travelling through potential energy landscapes of disordered materials: the activationrelaxation technique," Phys. Rev. E 57, 2419-2424 (1998).

19. G. Wei, N. Mousseau, and P. Derreumaux, "Exploring the energy landscape of proteins: a characterization of the activation relaxation technique," J. Chem. Phys. 117, 11379-11387 (2002).

20. CODE v, Optical Research Associates, Pasadena, Calif.

21. M. F. Bal, F. Bociort, and J. J. M. Braat, "Lithographic apparatus, device manufacturing method, and device manufactured thereby," U.S. patent 6,556,648 (29 April 2003).

22. D. M. Williamson, "High numerical aperture ring field optical reduction system," U.S. patent 5,815,310 (29 September 1998).

23. R. Hudyma, "High numerical aperture ring field projection system for extreme ultraviolet lithography," U.S. patent 6,183,095 (6 February 2001).

24. D. R. Shafer, "Projection lithography system and method using all-reflective optical elements," U.S. patent 5,686,728 (11 November 1997)

25. J. J. M. Braat, "Mirror projection system for a scanning lithographic projection apparatus, and lithographic apparatus comprising such a system," U.S. patent 6,299,318 (9 October 2001).

26. J. J. M. Braat, "Mirror projection system for a scanning lithographic projection apparatus, and lithographic apparatus comprising such a system," U.S. patent 6,255,661 (3 July 2001).

27. J. J. M. Braat, "Mirror projection system for a scanning lithographic projection apparatus, and lithographic apparatus comprising such a system," U.S. patent $6,396,067$ (28 May 2002). 
28. R. Hudyma, "High numerical aperture ring field projection system for extreme ultraviolet lithography," U.S. patent 6,033,079 (7 March 2000).

29. W. Ulrich, "8-mirror microlithography projection optics," U.S. patent 6,710,917 B2 (23 March 2004).

30. O. Marinescu, F. Bociort, and J. Braat, "Avoiding unstable regions in the design space of EUV mirror systems comprising high-order aspheric surfaces," Proc. SPIE 5523, 185-192 (2004) to be published in an updated version.

31. M. Isshiki, L. Gardner, and G. G. Gregory, "Automated control of manufacturing sensitivity during optimization," Proc. SPIE 5249, 343-352 (2004).

32. O. E. Marinescu, "Novel design methods for high-quality lithographic objectives," thesis, Delft University of Technology, Sieca, ISBN-13: 978-90-78314-02-8 (2006), also available at: http://wwwoptica.tn.tudelft.nl/publications/Thesis/ Marinescu.pdf.

33. M. F. Bal, F. Bociort, and J. J. M. Braat, "Analysis, search and classification for reflective ring-field projection systems," Appl. Opt. 42, 2301-2311 (2003).

34. H. Meiling, J. Benschop, U. Dinger, and P. Kurz, "Progress of the EUVL alpha tool," Proc. SPIE 4343, 38-50 (2001).

35. N. Harned, L. Girard, P. Kuerz, and L. Marchetti, "State of the art optics polishing and metrology for EUV lithography mirrors," http://www.asml.com/asmldotcom/show.do?ctx= $11140 \&$ rid $=11141$ 